Rev. Int. Contam. Ambie. 35 (1) 115-124, 2019

DOI: 10.20937/RICA.2019.35.01.08

\title{
ACETILCOLINESTERASA DE Eisenia foetida COMO INDICADOR DE CONTAMINACIÓN POR PLAGUICIDAS ORGANOFOSFORADOS
}

\author{
Magdalena GALINDO-GUZMÁN ${ }^{1}$, Erika FLORES-LOYOLA ${ }^{2 *}$, Miguel Ángel GALLEGOS-ROBLES ${ }^{1}$, \\ Manuel FORTIS-HERNÁNDEZ ${ }^{3}$, Uriel FIGUEROA-VIRAMONTES ${ }^{4}$ y Cirilo VÁZQUEZ-VÁZQUEZ ${ }^{1}$
}

${ }^{1}$ Facultad de Agricultura y Zootecnia, Universidad Juárez del Estado de Durango, km 35 carretera Gómez Palacio-Tlahualilo, Ejido Venecia, 35110 Gómez Palacio, Durango, México

${ }^{2}$ Facultad de Ciencias Biológicas, Universidad Autónoma de Coahuila. km 7.5 carretera Torreón-Matamoros, Ciudad Universitaria, 27276 Torreón, Coahuila, México

${ }^{3}$ Instituto Tecnológico de Torreón, División de Estudios de Posgrado, km 7.5 carretera Torreón-San Pedro, Ejido Ana, 27170 Torreón, Coahuila, México

${ }^{4}$ INIFAP, Campo Experimental La Laguna, Blvd. José Santos Valdés 1200 Poniente, 27440 Matamoros, Coahuila, México

*Autora para correspondencia: erika-flores@uadec.edu.mx

(Recibido febrero 2017; aceptado mayo 2018)

Palabras clave: inhibición enzimática, acetato de indoxilo, actividad enzimática, inhibidores organofosforados, selectividad

\section{RESUMEN}

Entre los plaguicidas inhibidores de la acetilcolinesterasa más usados en la actualidad, y a su vez los más tóxicos, se encuentran los organofosforados. La enzima acetilcolinesterasa (AChE) es ampliamente utilizada para la detección de contaminantes organofosforados, ya que éstos son inhibidores de su actividad. La lombriz de tierra (Eisenia foetida) es una fuente de esta enzima, ya que las AChE presentes en estas lombrices son susceptibles a dicho efecto. En este trabajo se preparó un concentrado enzimático de acetilcolinesterasa de $E$. foetida, con el cual se evaluó el efecto inhibitorio de plaguicidas organofosforados comerciales como malatión, diazinón, metamidofos y dimetoato, así como su selectividad al utilizar plaguicidas organoclorados, carbamatos y piretroides. La actividad de acetilcolinesterasa se midió usando acetato de indoxilo como sustrato cromogénico. Los resultados mostraron que todos los plaguicidas organofosforados ensayados en concentraciones de 1 a $10 \mathrm{mg} / \mathrm{L}$ fueron capaces de disminuir la actividad de acetilcolinesterasa en porcentajes de $36 \pm 16 \%$ en promedio, mientras que los carbamatos inhibieron sólo ligeramente la actividad enzimática en un $16 \pm 7 \%$. Los organoclorados y piretroides por su parte presentaron efecto inhibitorio poco o no significativo de $1 \pm 5 \%$ para cualquiera de las concentraciones ensayadas debido a que el modo de acción de los grupos de plaguicidas es diferente. Por este motivo, la actividad de la enzima no se ve afectada de manera significativa y continúa catalizando la descomposición del acetato de indoxilo aun en presencia de plaguicidas con grupos químicos diferentes a los organofosforados, lo cual obedece a la selectividad de la acetilcolinesterasa frente a este tipo de inhibidores.

Key words: enzymatic inhibition, indoxyl acetate, enzymatic activity, organophosphorus inhibitors, selectivity 


\begin{abstract}
Organophosphates are among the most used and the most toxic pesticides that inhibit acetylcholinesterase. The enzyme aceticolinesterase (AChE) is widely used to detect organophosphate pollutants because they are inhibitors of its activity. A source of this enzyme is present in Eisenia foetida, being the AChEs of these earthworms susceptible to this effect. In this work, an enzymatic concentrate of acetylcholinesterase was prepared from E. foetida. The inhibitory effect of commercial organophosphorus pesticides as malathion, diazinon, methamidophos and dimethoate on its enzymatic activity was evaluated, as well as its selectivity when using organochlorine, carbamate and pyrethroid pesticides. Acetylcholinesterase activity was measured using indoxyl acetate as chromogenic substrate. Results showed that all organophosphorus pesticides tested, in concentrations between $1-10 \mathrm{mg} / \mathrm{L}$, were able to decrease acetylcholinesterase activity in percentages of $36 \pm 16 \%$ on average. On the other hand, carbamates slightly inhibited the enzymatic activity in a $16 \pm 7 \%$, and organochlorines and pyrethroids showed a little or no significant inhibitory effect of $1 \pm 5 \%$ for any tested concentrations. This is because the mode of action of each group of pesticides is different. For this reason, the activity of the enzyme is not significantly affected and it remains catalyzing the decomposition of indoxyl acetate even in the presence of pesticides with chemical groups different to organophosphates, which is indicative of acetylcholinesterase selectivity against this specific type of inhibitors.
\end{abstract}

\section{INTRODUCCIÓN}

Entre las diversas clases de plaguicidas los organofosforados son los más empleados en la agricultura (Sharma et al. 2010), y son ampliamente utilizados como insecticidas debido a su alta actividad, baja bioacumulación y degradación moderadamente rápida en el ambiente.

Sin embargo, una cierta cantidad de los plaguicidas utilizados se transfiere en el escurrimiento superficial y subsuperficial del drenaje en tierras agrícolas y puede causar una variedad de efectos tóxicos en organismos acuáticos y seres humanos (Turdean et al. 2002, Kilany et al. 2014).

$\mathrm{Su}$ toxicidad recae principalmente en el sistema nervioso, inhibiendo la enzima acetilcolinesterasa (AChE). Esto produce una acumulación de acetilcolina en las sinapsis nerviosas y una alteración de la función nerviosa, siendo las AChE de insectos especialmente susceptibles a este efecto (Constantine et al. 2003, Lang et al. 2016).

La acetilcolinesterasa es una enzima del grupo de las hidrolasas que está presente en la mayoría de los seres vivos, principalmente en los vertebrados, incluyendo a los seres humanos, pero también en invertebrados, como los insectos.

La AChE se localiza en el sistema nervioso y los músculos, y es la responsable de la regulación de la concentración de acetilcolina (AC), un neurotransmisor involucrado en la sinapsis colinérgica en el sistema nervioso que permite la transmisión de señales en el sistema nervioso central y periférico (Wiesner et al. 2007).

Los plaguicidas organofosforados se unen a la enzima AChE e inhiben su actividad por fosforilación irreversible, por lo cual su restauración depende de la síntesis de nuevas moléculas de esta enzima (Cárdenas et al. 2010). La inhibición de la actividad AChE por muchos plaguicidas organofosforados (OF) está bien establecida en diferentes especies animales (Venkateswara y Kavitha 2004), tanto en condiciones de laboratorio como de campo (Chakra y Venkateswara 2008).

Las lombrices de tierra están actualmente consideradas como una alerta temprana sobre los efectos negativos de los plaguicidas (Gambi 2007), principalmente de los organofosforados, los cuales son conocidos como agentes neurotóxicos que causan toxicidad aguda en estos organismos (Scott-Fordsmand y Weeks 2000, Venkateswara et al. 2003).

De acuerdo con lo anterior, se puede establecer que a través del ensayo de la actividad AChE es posible realizar el diagnóstico de la exposición a plaguicidas organofosforados (Bajgar 2004, Pohanka et al. 2011). Por ello en este trabajo se presenta una estrategia para determinar el ensayo de la actividad AChE de lombriz de tierra (Eisenia foetida) utilizando acetato de indoxilo como alternativa de sustrato cromogénico (Villatte et al. 2001). 
El principio de reacción de este sustrato se muestra en la figura 1, donde se observa que la hidrólisis del acetato de indoxilo (incoloro) es catalizada por la AChE produciendo indoxilo, el cual se oxida rápidamente y genera un color azul índigo que puede distinguirse bien a simple vista. La intensidad del color es indicativa de la actividad AChE, por lo tanto el grado de inhibición enzimática generado por la contaminación de residuos de plaguicidas organofosforados puede determinarse por este método (Guo et al. 2013).

\section{MATERIALES Y MÉTODOS}

\section{Extracción enzimática de acetilcolinesterasa}

Veinticinco gramos de lombrices de tierra (Eisenia foetida) fueron homogeneizados en $75 \mathrm{~mL}$ de una solución amortiguadora de $\mathrm{NaH}_{2} \mathrm{PO}_{4} 0.02 \mathrm{M}$, tritón $\times 1000.1 \%$ y pH 7. El material homogeneizado se centrifugó durante $25 \mathrm{~min}$ a $10000 \mathrm{~g} \mathrm{a} 4{ }^{\circ} \mathrm{C}$. Los sobrenadantes fueron recuperados para obtener un extracto enzimático crudo (Schreck 2009), el cual fue liofilizado y almacenado a $4{ }^{\circ} \mathrm{C}$ para realizar los ensayos enzimáticos.

La estimación de proteínas se cuantificó con el método de Bradford (1976) y la actividad se calculó utilizando el coeficiente de extinción molar del acetato de indoxilo $\left(\varepsilon=3900 \mathrm{M}^{-1} \mathrm{~cm}^{-1}\right)$ reportado en la literatura (Pohanka et al. 2011).

\section{Ensayo de la actividad AChE utilizando acetato de indoxilo}

Para cada ensayo enzimático se mezclaron $5 \mathrm{mg}$ de enzima AChE, $1.4 \mathrm{~mL}$ de amortiguador de fosfato de sodio $(0.1 \mathrm{M})$ y $100 \mu \mathrm{L}$ de acetato de indoxilo $(0.05 \mathrm{M})$ en metanol al $5 \%$. Esta concentración de metanol fue seleccionada para evitar la inhibición de AChE y permitir la solubilidad del sustrato (Pohanka et al. 2009).

La mezcla fue agitada y después de $10 \mathrm{~min}$ se midió su absorbancia a una longitud de onda de 670 $\mathrm{nm}$ en un espectrofotómetro ultravioleta-visible Genesys 10UV Scanning (Thermo Scientific) (Pohanka et al. 2011). La actividad enzimática se reportó mediante el estudio de la variación de la densidad óptica (DO).

\section{Inhibición enzimática por plaguicidas}

Para la determinación del efecto de los plaguicidas sobre la actividad AChE, se incubaron $5 \mathrm{mg}$ de enzima con $1 \mathrm{~mL}$ de cada plaguicida diluido en concentraciones de 1 a $10 \mathrm{mg} / \mathrm{L}$ a $25^{\circ} \mathrm{C}$ durante 20 min; posteriormente se añadieron $0.4 \mathrm{~mL}$ de amortiguador de fosfato de sodio $0.1 \mathrm{My} 100 \mu \mathrm{L}$ de acetato de indoxilo $0.05 \mathrm{M}$ en metanol al $5 \%$ en agua. La absorbancia de la mezcla se midió 20 min después a la longitud de onda establecida. Este proceso fue realizado por triplicado.

El grado de inhibición se calculó mediante la siguiente ecuación (Arduini et al. 2005):

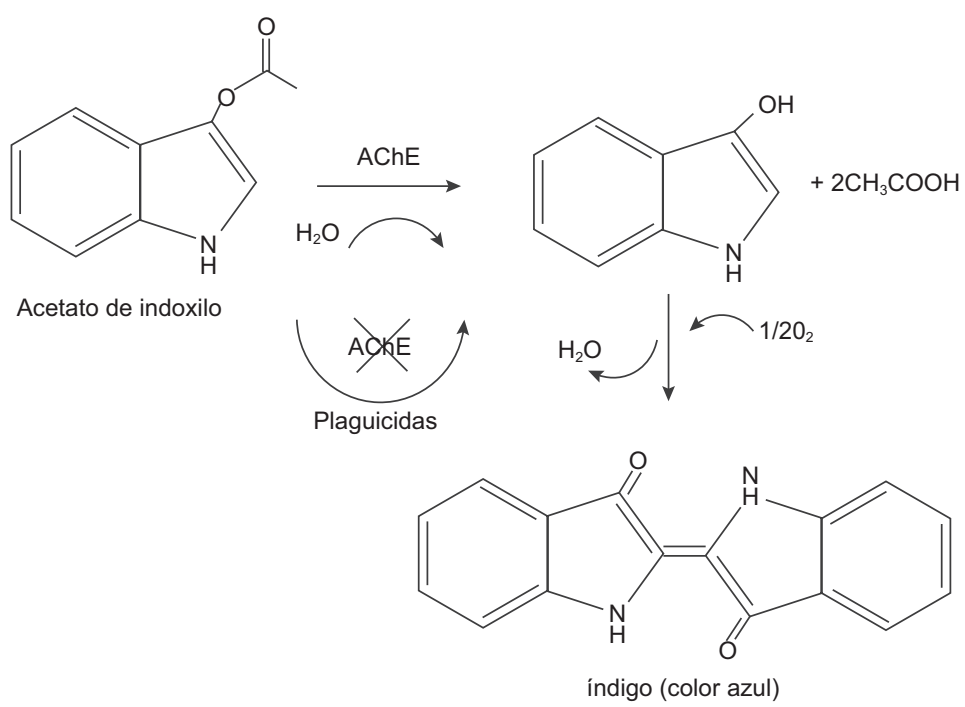

Fig. 1. Mecanismo de hidrólisis del acetato de indoxilo catalizada por AChE (Apilux et al. 2015) 
$I \%=\left(A_{0}-A_{\mathrm{i}}\right) / A_{0}$

donde $I \%$ es el porcentaje de inhibición, $A_{0}$ es la absorbancia de la solución enzimática con el acetato de indoxilo sin contaminante y $A_{\mathrm{i}}$ es la absorbancia de la mezcla de AChE y acetato de indoxilo con el plaguicida.

Los plaguicidas estudiados fueron de tipo comercial: cuatro organofosforados (malatión, diazinón, dimetoato, metamidofos), un carbamato (carbofurano), un piretroide (cipermetrina) y un organoclorado (endosulfán). Esto con el fin de evaluar el efecto de los plaguicidas con diferentes grupos químicos en la inhibición de la AChE y por lo tanto determinar su selectividad.

El análisis experimental se realizó bajo un diseño de bloques completamente al azar con tres repeticiones, y la comparación de medias se realizó por el método de la diferencia mínima significativa (DMS) al 0.05 de probabilidad.

\section{RESULTADOS}

\section{Optimización de las condiciones experimentales}

La actividad enzimática del extracto de $\mathrm{AChE}$ fue evaluada usando diferentes concentraciones de sustrato (0.01-0.10 M) con el fin de determinar las condiciones óptimas de hidrólisis del acetato de indoxilo por esta enzima. Los resultados obtenidos se muestran en la figura 2a. Como era de esperarse, la intensidad del color generado por la formación de indoxilo aumentó con el incremento de la concentración del sustrato. De acuerdo con lo anterior, para las siguientes pruebas la concentración de sustrato utilizada fue de $0.05 \mathrm{M}$, ya que, como se muestra en la figura 2a, con esta concentración se obtiene el máximo de actividad de la enzima sin comprometer la competencia entre plaguicidas por exceso de sustrato.

En la figura $2 \mathbf{b}$ se presenta el curso temporal de la reacción de $\mathrm{AChE}$ en los preparados de liofilizado de E. foetida a $25^{\circ} \mathrm{C}$. Se observa que la hidrólisis del
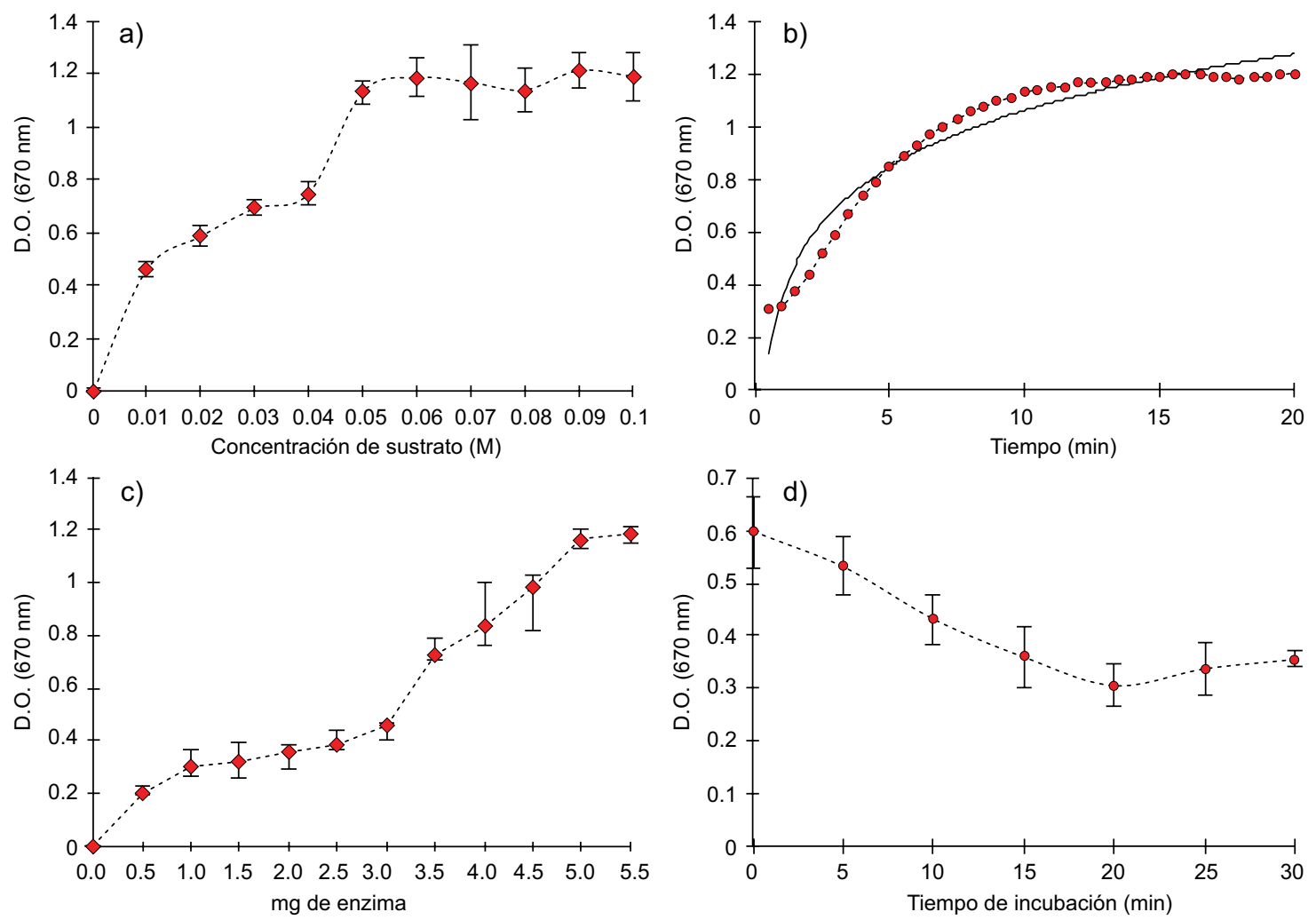

Fig. 2. Optimización de las condiciones experimentales. (a) Efecto de la concentración de sustrato (acetato de indoxilo) sobre la actividad enzimática de AChE. (b) Curva de desarrollo de la reacción enzimática de AChE. (c) Actividad catalítica en unidades de absorbancia (DO) determinada con diferentes cantidades de enzima. (d) Tiempo de incubación del malatión (representante seleccionado de plaguicidas concentración de $10 \mathrm{mg} / \mathrm{L}$ ). Las barras de error indican la desviación estándar para $n=3$ 
sustrato por la enzima mostró un comportamiento tipo Michaelis-Menten y que la actividad fue en aumento hasta el final de la incubación.

La determinación de la dependencia de la actividad enzimática respecto a la cantidad de AChE utilizada se muestra en la figura 2c. Como puede observarse, el mayor valor de la actividad ocurre cuando se adicionan entre 5 y $5.5 \mathrm{mg}$ de liofilizado enzimático, por lo cual $5 \mathrm{mg}$ fue la cantidad utilizada en experimentos posteriores.

El resultado de la evaluación de la dependencia del tiempo de incubación del inhibidor con la AChE utilizando malatión se muestra en la figura 2d. En dicha figura se observa que hay una reducción de la DO a medida que el tiempo se incrementa; sin embargo, después de 20 min esta reducción ya no es significativa. Por lo anterior, en tratamientos subsiguientes la solución de plaguicida se incubó con la AChE durante 20 min a temperatura ambiente antes de realizar el ensayo enzimático con el sustrato.

Una vez determinados los parámetros adecuados para los ensayos enzimáticos de la acetilcolinesterasa extraída de E. foetida, se determinó su actividad específica, siendo ésta de $0.00854 \mathrm{U} / \mathrm{mg}$ de proteína.

Con el fin de evaluar el efecto de los plaguicidas sobre la enzima AChE se realizó un análisis de varianza para el porcentaje de inhibición enzimática (Cuadro I), el cual muestra que hubo diferencias altamente significativas $(\mathrm{p}<0.01)$ para los tres factores de estudio y sus interacciones entre grupos químicos, plaguicidas y concentraciones.

CUADRO I. ANÁLISIS DE VARIANZA PARA EL PORCENTAJE DE INHIBICIÓN ENZIMÁTICA ENTRE GRUPOS QUÍMICOS, PLAGUICIDAS Y CONCENTRACIONES

\begin{tabular}{lrc}
\hline FV & CM & Pr $>$ F \\
\hline Repeticiones & 13.26 & 0.649 \\
Grupo químico (G) & 18879.28 & $<.0001$ \\
Plaguicida (P) & 10239.97 & $<.0001$ \\
$\mathrm{G} \times \mathrm{P}$ & 872.23 & $<.0001$ \\
Concentración (C) & 1574.27 & $<.0001$ \\
$\mathrm{G} \times \mathrm{C}$ & 234.12 & $<.0001$ \\
$\mathrm{P} \times \mathrm{C}$ & 155.98 & $<.0001$ \\
$\mathrm{G} \times \mathrm{P} \times \mathrm{C}$ & 2783.87 & $<.0001$ \\
Error & 30.59 & \\
\hline
\end{tabular}

\section{Inhibición enzimática por plaguicidas organo- fosforados}

Se evaluó el efecto in vitro de los plaguicidas pertenecientes al grupo de los OF, usando concentraciones entre 1-10 mg/L, sobre la actividad de la enzima AChE. Los resultados de la comparación de medias del porcentaje de inhibición enzimática (Fig. 3) mostraron que la intensidad de color del producto de la reacción enzimática disminuye con el aumento de la concentración de plaguicida. Esto establece que todos los plaguicidas organofosforados ensayados (malatión, metamidofos, diazinón, dimetoato) tienen la capacidad de reducir esta actividad desde un $29 \pm$ $11 \%$ hasta un $48 \pm 12 \%$ en intervalos de concentraciones de 1 a $10 \mathrm{mg} / \mathrm{L}$. Es importante señalar que sólo la muestra de referencia sin plaguicidas dio valores de porcentaje de inhibición significativamente diferentes del resto de las muestras.

\section{Porcentaje de inhibición de la actividad AChE por plaguicidas}

La comparación de medias del porcentaje de inhibición enzimática generada por los diferentes plaguicidas utilizando concentraciones de 1 a $10 \mathrm{mg} / \mathrm{L}$ se muestra en la figura 4, donde se observa que una inhibición significativa se llevó a cabo en presencia de plaguicidas como dimetoato $(43 \pm 15 \%)$, diazinón $(39 \pm 15 \%)$, malatión $(37 \pm 17 \%)$ y metamidofos $(27$ $\pm 10 \%)$, y en menor grado carbofurano $(16 \pm 7 \%)$, en tanto que endosulfán $(1 \pm 5 \%)$ y cipermetrina $(0.9$ $\pm 4 \%$ ) presentaron poco o ningún efecto inhibitorio significativo para cualquiera de las concentraciones ensayadas.

\section{Selectividad en la inhibición de $\mathrm{AChE}$}

Otros experimentos se llevaron a cabo en presencia de plaguicidas con grupos químicos diferentes utilizando concentraciones de 1 a $10 \mathrm{mg} / \mathrm{L}$, con el fin de determinar la inhibición selectiva de la AChE por plaguicidas organofosforados. Los resultados (Fig. 5) muestran que hubo inhibición significativa en presencia de plaguicidas organofosforados $(36 \pm 16 \%)$ y carbamatos $(16 \pm 7 \%)$, mientras que los organoclorados y piretroides presentaron poco o ningún efecto inhibitorio significativo $(1 \pm 5 \%)$, independientemente de la concentración.

\section{DISCUSIÓN}

Diversos factores afectan la sensibilidad del ensayo de actividad enzimática de $\mathrm{AChE}$, los cuales incluyen la cantidad de enzima, la concentración del sustrato, el tiempo de incubación y la presencia de plaguicidas (Apilux et al. 2015). Por lo tanto, se determinaron las condiciones óptimas para el análisis de plaguicidas. 


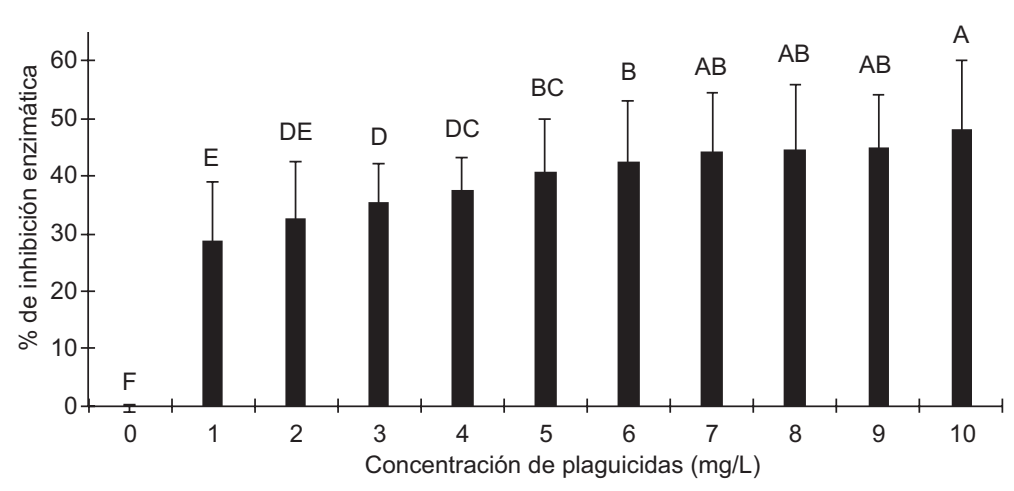

Fig. 3. Comparación de medias del porcentaje de inhibición enzimática de acetilcolinesterasa de lombriz (Eisenia foetida) con diferentes concentraciones de plaguicidas organofosforados. Valores con letras distintas son estadísticamente significativos $(\mathrm{p}<0.05)$

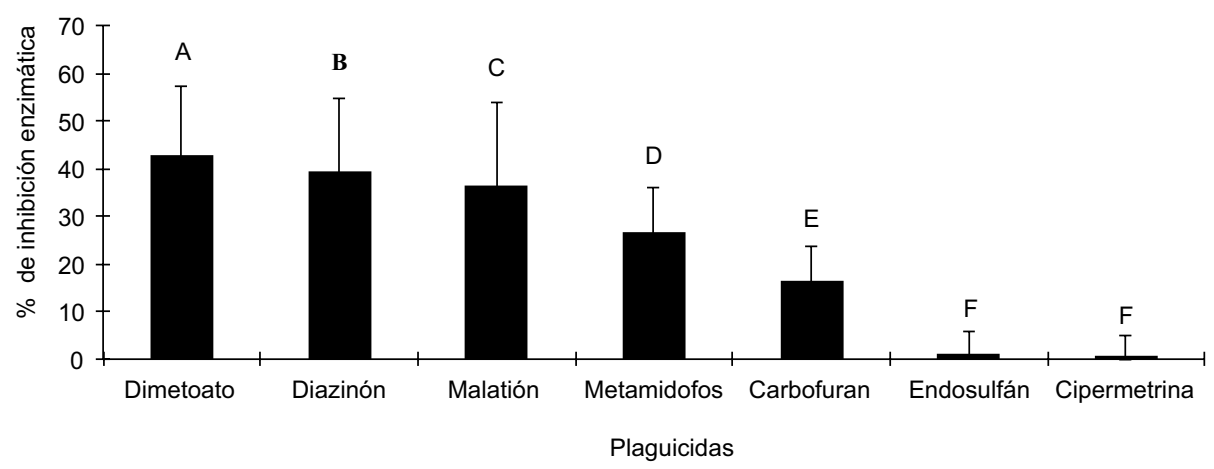

Fig. 4. Comparación de medias del porcentaje de inhibición enzimática de acetilcolinesterasa de lombriz (Eisenia foetida) entre los diferentes plaguicidas. Valores con letras distintas son estadísticamente significativos $(\mathrm{p}<0.05)$

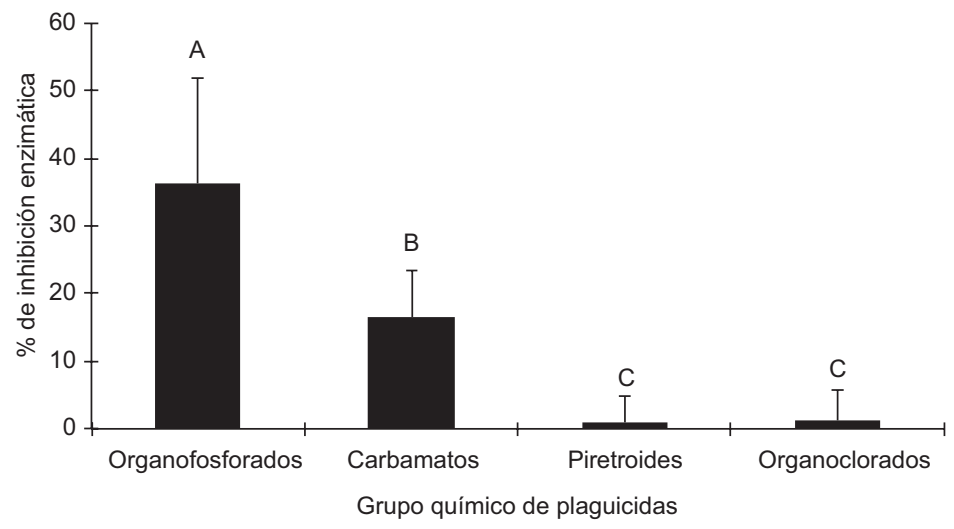

Fig. 5. Comparación de medias del porcentaje de inhibición enzimática de acetilcolinesterasa de lombriz (Eisenia foetida) con diferentes grupos químicos de plaguicidas. Valores con letras distintas son estadísticamente significativos $(\mathrm{p}<0.05)$ 
En ensayos de concentración de acetato de indoxilo no se observó inhibición por sustrato en concentraciones de hasta $0.1 \mathrm{M}$ (Fig. 2a). Esto puede deberse a que el acetato de indoxilo no es un compuesto altamente soluble y aunque se encuentre en exceso no interacciona con la enzima en el medio de reacción utilizado (Pohanka et al. 2011).

Los ensayos in vitro demostraron que el acetato de indoxilo es un sustrato cromogénico adecuado para la evaluación de la actividad AChE (Pohanka y Drtinova 2013, Pohanka y Vlcek 2014). Esto obedece a la rápida formación de coloración azul índigo debida al indoxilo producido por reacción del sustrato con la enzima, de acuerdo con el mecanismo de reacción mostrado en la figura 1 (Pohanka y Vlcek 2014).

Respecto del seguimiento de la formación de producto en función del tiempo, en la figura $\mathbf{2 b}$ se observa que después de 10 min de reacción se alcanza la máxima concentración de indoxilo. Pasado este tiempo no se observa incremento en la concentración de producto, proceso generalmente debido al consumo de sustrato disponible para la reacción.

La dependencia de la actividad enzimática con relación a la concentración de extracto enzimático (Fig. 2c) muestra que la cantidad de producto aumenta con el incremento de la cantidad de catalizador (Apilux et al. 2015). Es decir, la cantidad de catalizador limita la velocidad de la reacción; sin embargo, cantidades mayores de $5 \mathrm{mg}$ de extracto enzimático liofilizado no generan incrementos importantes en la velocidad de reacción.

En la figura 2d se muestra que 20 min de incubación del complejo enzima-plaguicida son suficientes para que los plaguicidas como malatión interaccionen con la enzima inhibiendo su actividad y confirmando que en el medio existe una sustancia tóxica que afecta a la AChE.

La significancia observada en el análisis de varianza para los tres factores de estudio indica que el grado de inhibición enzimática depende del grupo químico y el tipo del plaguicida, así como de la concentración del ingrediente activo. Esto quiere decir que, aun cuando pertenezcan a un mismo grupo químico, los plaguicidas comerciales tienen una respuesta diferente en la inhibición enzimática. Esto se debe a que la reactividad de los compuestos organofosforados varía de acuerdo con su estructura química, principalmente por la distribución de los átomos unidos al fósforo, por ejemplo, los que tienen un doble enlace entre fósforo y oxígeno son altamente electrofílicos y altamente reactivos (Mileson et al. 1998). Entre grupos químicos la significancia observada fue la esperada, ya que los grupos piretroides y organoclorados no tienen un efecto inhibitorio sobre la AChE (Ibrahim et al. 1998). La significancia observada entre las diferentes concentraciones también es un indicador de que la actividad enzimática disminuye con el aumento de la dosis de plaguicidas (Ibrahim et al. 1998).

\section{Inhibición enzimática por plaguicidas organo- fosforados}

La actividad de la AChE en presencia de plaguicidas OF se reduce debido al tipo de interacción enzima-sustrato (Fig. 3). En contacto con un compuesto organofosforado, esta enzima sufre un proceso de fosforilación mediante la producción de un complejo estable en su sitio activo, lo que altera su actividad catalítica (Dzudzevic et al. 2016).

Estos compuestos interaccionan con serina en la triada catalítica del sitio activo, formando grupos de ésteres estables (Pohanka 2011). Después de un intervalo de tiempo específico para cada inhibidor OF, el inhibidor unido sufre una reacción de desalquilación interna llamada "envejecimiento" (Millard et al. 1999) para producir un complejo conjugado de OF-enzima que no puede ser reactivado, generando así una inhibición irreversible.

El envejecimiento no tiene ningún efecto beneficioso sobre la enzima, ya que después de este permanece inactiva, lo que sugiere que las fuerzas electrostáticas estabilizan significativamente a la enzima envejecida (Pohanka 2011). Este efecto visibiliza en los resultados que muestran que la actividad enzimática disminuye con el aumento de la concentración de plaguicida (Apilux et al. 2015).

La inhibición de la AChE por plaguicidas OF indica que este ensayo puede utilizarse para la confirmación de la presencia de estos contaminantes en un medio determinado según las concentraciones del estudio. Todas las muestras contaminadas por plaguicidas OF son similares entre sí, ya que en todos los casos existe un grado de inhibición enzimática semejante, lo cual indica que concentraciones tan bajas como $1 \mathrm{mg} / \mathrm{L}$ pueden detectarse por este método. Lo anterior demuestra la alta sensibilidad de los ensayos enzimáticos para este tipo de análisis.

De acuerdo con el análisis de comparación de medias puede observarse que la muestra de agua utilizada como control es estadísticamente diferente a las muestras contaminadas, lo cual se debe a que en esta muestra no se presenta inhibición de la AChE. Lo anterior indica que es posible diferenciar muestras libres de contaminantes organofosforados de aquellas que los contienen en concentraciones de 1 a $10 \mathrm{mg} / \mathrm{L}$. 


\section{Porcentaje de inhibición de plaguicidas}

Los resultados de la comparación de medias del porcentaje de inhibición para todos los plaguicidas utilizados (Fig. 4) muestran que el biocatalizador de AChE es más sensible a la presencia de plaguicidas organofosforados como dimetoato, diazinón, malatión y metamidofos (porcentaje de inhibición significativa más pronunciada).

En el estudio también se utilizó carbofurano, un carbamato que se emplea comúnmente en todo el mundo como insecticida, nematicida y acaricida en la práctica agrícola (Gupta 1994). Se trata de uno de los plaguicidas más tóxicos en la agricultura y su dosis letal para mamíferos se encuentra entre los 5 y $50 \mathrm{mg} / \mathrm{kg}$ (Jeyapragasam y Saraswathi 2014). Al igual que los plaguicidas organofosforados, la inhibición enzimática para carbofurano también es significativa, aunque ocurre en menor grado (Ibrahim et al. 1998).

Por otro lado, cuando la $\mathrm{AChE}$ es expuesta a plaguicidas como endosulfán y cipermetrina no se observan efectos negativos significantivos sobre la actividad enzimática en concentraciones de 1 a $10 \mathrm{mg} / \mathrm{L}$.

Los resultados del porcentaje de inhibición obtenidos en este estudio son similares a los reportados por el análisis de plaguicidas utilizando AChE extraída de otros organismos como Chironomus riparius meigen. Tal es el caso reportado por Ibrahim et al. (1998), quienes observaron un rango del 20 al $50 \%$ de inhibición por plaguicidas organofosforados y carbamatos en concentraciones de $0.19-0.84 \mu \mathrm{M}$ y 0.06-0.36 $\mu \mathrm{M}$, respectivamente.

\section{Selectividad en la inhibición de AChE}

El grupo químico de los plaguicidas organoclorados como el endosulfán y el grupo de los piretroides como cipermetrina (Fig. 5) no presentaron ningún efecto inhibitorio sobre la actividad AChE, debido a que su modo de acción dos difiere del de los organofosforados (Devine et al. 2008).

Los plaguicidas organoclorados son antagonistas del canal de cloruro regulado por el ácido gammaaminobutírico (GABA) e interfieren con los canales de cloruro en la membrana nerviosa (Pomés et al. 1994). Los plaguicidas piretroides, por su parte, actúan como moduladores del canal de sodio e interfieren con dichos canales en la membrana nerviosa (Soderlund 2012).

Lo anterior confirma la especificidad de la AChE, motivo por el cual la actividad de la enzima no se ve afectada de manera significativa y continúa catalizando la descomposición de acetato de indoxilo aun en presencia de estos plaguicidas. Lo anterior coincide con lo establecido por Ibrahim et al. (1998), quienes incubaron $\mathrm{AChE}$ in vitro extraída de Chironomus riparius meigen en presencia de plaguicidas con grupos químicos organoclorados y piretroides, $\mathrm{y}$ demostraron que no hay efecto sobre la actividad de la AChE por estos compuestos.

Por otro lado, los carbamatos como el carbofurano son compuestos orgánicos derivados de ésteres del ácido carbámico, y al igual que los OF inhiben la actividad $\mathrm{AChE}$ (Fig. 5). Aunque los compuestos organofosforados y carbamatos pertenecen a grupos químicos diferentes, el mecanismo a través del cual producen toxicidad es similar. Como ya se mencionó, este mecanismo se relaciona con la inhibición de AChE que da lugar a acumulación de acetilcolina, lo cual altera el funcionamiento normal de los impulsos nerviosos e interfiere con la sinapsis. El grupo funcional éster de los plaguicidas organofosforados y los carbamatos podría ser, teóricamente, hidrolizado por estas enzimas (Ménendez 2009). En comparación con los inhibidores organofosforados, el resto del carbamato se hidroliza espontáneamente y se libera, por lo que la AChE puede reactivarse. Esto sugiere que los carbamatos tienen probablemente la capacidad de unirse a través de interacciones no covalentes (Pohanka 2011), lo cual explica el hecho de que provoquen una inhibición de la $\mathrm{AChE}$, pero no tan fuerte como la generada por los organofosforados.

\section{CONCLUSIONES}

Los plaguicidas organofosforados y carbamatos se caracterizan por su capacidad para inhibir la enzima AChE de manera selectiva, por lo que la medición de la actividad enzimática puede proporcionar información fiable para monitorizar posibles exposiciones a estos contaminantes.

Con base en los resultados se estableció que el sistema catalítico del extracto crudo de AChE de Eisenia foetida permite detectar la presencia o ausencia exclusiva de plaguicidas organofosforados inhibidores de esta enzima. Plaguicidas como endosulfán y cipermetrina no muestran inhibición aparente debido a las diferencias en su modo de acción.

El ensayo para monitorear plaguicidas in vitro es una alternativa de análisis de bajo costo, con un consumo mínimo de reactivos y no requiere de personal altamente capacitado. El acetato de indoxilo como sustrato reactivo cromogénico es adecuado para la evaluación de este mecanismo de reacción y puede ser de gran utilidad como apoyo para el desarrollo de nuevas tecnologías de detección que sugieran la posible presencia de plaguicidas en concentraciones tan bajas como $1 \mathrm{mg} / \mathrm{L}$. 


\section{REFERENCIAS}

Apilux A., Isarankura-Na-Ayudhya C., Tantimongcolwat T. y Prachayasittikul V. (2015). Paper-based acetylcholinesterase inhibition assay combining a wet system for organophosphate and carbamate pesticides detection. EXCLI J. 14, 307-319. DOI: 10.17179/excli2014-684

Arduini F., Ricci F., Bourais I., Amine A., Moscone D. y Palleschi G. (2005). Extraction and detection of pesticides by cholinesterase inhibition in a two-phase system: A strategy to avoid heavy metal interference. Anal. Lett. 38 (11), 1703-1719.

DOI: $10.1080 / 00032710500206970$

Bajgar J. (2004). Organophosphates/nerve agent poisoning: Mechanism of action, diagnosis, prophylaxis, and treatment. Adv. Clin. Chem. 38, 151-216.

DOI: $10.1016 / \mathrm{S} 0065-2423(04) 38006-6$

Bradford M. (1976). A rapid and sensitive method for the quantitation of microgram quantities of protein utilizing the principle of protein dye binding. Anal. Biochem. 72 (1-2), 248-254.

DOI: 10.1016/0003-2697(76)90527-3

Cárdenas O., Silva E. y Ortiz J. (2010). Uso de plaguicidas inhibidores de acetilcolinesterasa en once entidades territoriales de salud en Colombia, 2002-2005. Biomédica 30 (1), 95-106. DOI: 10.7705/biomedica.v30i1.157

Chakra R. y Venkateswara R. (2008). Biological response of earthworm, Eisenia foetida (Savigny) to an organophosphorous pesticide, profenofos. Ecotoxicol. Environ. Saf. 71 (2), 574-582.

DOI: 10.1016/j.ecoenv.2008.01.003

Constantine C., Mello S., Dupont A., Cao X., Santos D., Oliveira O., Strixino F., Pereira E., Cheng T., Defrank J. y Leblanc R. (2003). Layer-by-layer self-assembled chitosan/poly(thiophene-3-acetic acid) and organophosphorus hydrolase multilayers. J. Am. Chem. Soc. 125 (7), 1805-1809.

DOI: $10.1021 / \mathrm{ja} 028691 \mathrm{~h}$

Devine G., Eza D., Ogusuku E. y Furlong M. (2008). Uso de insecticidas: contexto y consecuencias ecológicas. Rev. Peru. Med. Exp. Salud Pública 25 (1), 74-100.

Dzudzevic C., Soylemez S., Akpinar Y., Kesik M., Göker S., Gunbas G., Volkan M. y Toppare L. (2016). A novel acetylcholinesterase biosensor: Core-shell magnetic nanoparticles incorporating a conjugated polymer for the detection of organophosphorus pesticides. ACS Appl. Mater. Interfaces 8 (12), 8058-8067.

DOI: $10.1021 /$ acsami.5b12383

Gambi N., Pasteris A. y Fabbri E. (2007). Acetylcholinesterase activity in the earthworm Eisenia andrei at different conditions of carbaryl exposure. Comp. Biochem. Physiol. C 145 (4), 678-685.

DOI: $10.1016 / j . c b p c .2007 .03 .002$
Guo X., Zhang X., Cai Q., Shen T. y Zhu S. (2013). Developing a novel sensitive visual screening card for rapid detection of pesticide residues in food. Food Control 30 (1), 15-23. DOI: 10.1016/j.foodcont.2012.07.015

Gupta R. (1994). Carbofuran toxicity. J. Toxicol. Environ. Health 43 (4), 383-418. DOI: $10.1080 / 15287399409531931$

Ibrahim H., Kheir R., Helmi S., Lewis J. y Crane M. (1998). Effects of organophosphorus, carbamate, pyrethroid and organochlorine pesticides, and a heavy metal on survival and cholinesterase activity of Chironomus riparius meigen. Bull. Environ. Contam. Toxicol. 60 (3), 448-455.

DOI: $10.1007 / \mathrm{s} 001289900646$

Jeyapragasam T. y Saraswathi R. (2014). Electrochemical biosensing of carbofuran based onacetylcholinesterase immobilized onto iron oxide chitosannanocomposite. Sensor. Actuat. B-Chemical 191, 681-687. DOI: 10.1016/j.snb.2013.10.054

Kilany A., Elsayed M., Elmegid M. y Fayed M. (2014). Study of the effect of air to fuel ratio parameter on the organophosphorus-pesticide analysis by GC-FPD. ILCPA 17 (3), 236-248.

DOI: 10.18052/www.scipress.com/ILCPA.36.236

Lang Q., Han L., Hou C., Wang F. y Liu A. (2016). A sensitive acetylcholinesterase biosensor based on gold nanorods modified electrode for detection of organophosphate pesticide. Talanta 156-157, 34-41.

DOI: $10.1016 / j$.talanta.2016.05.002

Ménendez F. (2009). Higiene industrial. Manual para la formación del especialista. 9a ed. Lex Nova, Valladolid, España, 633 pp.

Mileson B., Chambers J., Chen W., Dettbarn W., Ehrich M., Eldefrawi A., y Padilla S. (1998). Common mechanism of toxicity: A case study of organophosphorus pesticides. Toxicol. Sci. 41 (1), 8-20.

DOI: $10.1093 /$ toxsci/41.1.8

Millard C., Kryger G., Ordentlich A., Greenblatt H., Harel M., Raves M., Segall Y., Barak D., Shafferman A., Silman I. y Sussman J. (1999). Crystal structures of aged phosphonylated acetylcholinesterase: Nerve agent reaction products at the atomic level. Biochemistry 38 (22), 7032-7039. DOI: 10.1021/bi9826781

Pohanka M., Dobes P., Drtinova L. y Kuca K. (2009). Nerve agents assay using cholinesterase based biosensor. Electroanalysis 21 (10), 1177-1182.

DOI: $10.1002 /$ elan.200804528

Pohanka M. (2011). Cholinesterases, a target of pharmacology and toxicology. Biomed. Pap. Olomouc. 155 (3), 219-230.

DOI: $10.5507 / \mathrm{bp} .2011 .036$

Pohanka M., Hrabinova M., Kuca K. y Simonato J.P. (2011). Assessment of acetylcholinesterase activity 
using indoxylacetate and comparison with the standard Ellman's method. Int. J. Mol. Sci. 12 (4), 2631-2640. DOI: $10.3390 /$ ijms 12042631

Pohanka M. y Drtinova L. (2013). Spectrophotometric methods based on 2,6-dichloroindophenol acetate and indoxylacetate for butyrylcholinesterase activity assay in plasma. Talanta 106, 281-285.

DOI: $10.1016 /$ j.talanta.2012.10.085

Pohanka M. y Vlcek V. (2014). Preparation and performance of a colorimetric biosensor using acetylcholinesterase and indoxylacetate for assay of nerve agents and drugs. Interdiscip. Toxicol. 7 (4), 215-218. DOI: 10.2478/intox-2014-0031

Pomés A., Rodríguez E. y Suñol C. (1994). Effects of organochlorine pesticides on $36 \mathrm{Cl}$ - flux in primary neuronal cultures. Neurotoxicology 15 (3), 745-749.

Schreck E., Geret F., Gontier L. y Treilhou M. (2009). ChE, GST and CAT: evaluation of the efficiency of a combined buffer for protein extraction. Ecotoxicol. Environ. Saf. 72 (5), 1609-1613.

DOI: 10.1016/j.ecoenv.2008.12.013

Scott-Fordsmand J.J., Weeks J.M. (2000) Biomarkers in earthworms. In: Reviews of environmental contamination and toxicology (Ware G.W., Ed.). Springer, New York, NY, USA, 117-159.

DOI: 10.1007/978-1-4612-1172-3_3

Sharma D., Nagpal A., Pakade Y. y Katnoria J. (2010). Analytical methods for estimation of organophosphorus pesticide residues in fruits and vegetables: A review. Talanta 82 (4), 1077-1089.

DOI: $10.1016 /$ j.talanta.2010.06.043
Soderlund D. (2012). Molecular mechanisms of pyrethroid insecticide neurotoxicity: Recent advances. Arch Toxicol. 86 (2), 165-181. DOI: 10.1007/s00204-011-0726-x

Turdean G., Popescu I., Oniciu L. y Thevenot D. (2002). Sensitive detection of organophosphorus pesticides using a needle type amperometric acetylcholinesterasebased bioelectrode. Thiocholine electrochemistry and immobilised enzyme inhibition. J. Enzyme Inhib. Med. Chem. 17 (2), 107-115.

DOI: $10.1080 / 14756360290026469$

Venkateswara R. y Kavitha P. (2004). Toxicity of azodrin on the morphology and acetylcholinesterase activity of the earthworm Eisenia foetida. Environ. Res. 96 (3), 323-327. DOI: 10.1016/j.envres.2004.02.014

Venkateswara R., Surya P. y Madhavendra S. (2003). Toxic effects of chlorpyrifos on survival, morphology and acetylcholinesterase activity of the earthworm Eisenia foetida. Ecotoxicol. Environ. Saf. 54, 296-301. DOI: 10.1016/S0147-6513(02)00013-1

Villatte F., Bachman T., Hussein A. y Schmid R. (2001). Acetylcholinesterase assay for rapid expression screening in liquid and solid media. Biotechniques 30 (1), 81-84. DOI: $10.2144 / 01301 \mathrm{st} 04$

Wiesner J., Kriz Z., Kuca K., Jun D. y Koca J. (2007). Acetylcholinesterases - the structural similarities and differences. J. Enzyme Inhib. Med. Chem. 22 (4), 417 424. DOI: $10.1080 / 14756360701421294$ 